\title{
Expression anormale du proto-oncogène c-fos dans l'os atteint de dysplasie fibreuse
}

La dysplasie fibreuse est une maladie sporadique affectant le développement osseux. Les patients atteints montrent des lésions caractérisées par une fibrose de la moelle, un remodelage accéléré et la persistance d'os tissé, rappelant l'os embryonnaire. Ces anomalies de la structure de l'os, qui peuvent être localisées à un site unique (dysplasie fibreuse monostotique) ou affecter tout le squelette (dysplasie fibreuse polyostotique), sont responsables des fractures et déformations dont souffrent les patients. Lorsqu'elle est associée à une puberté précoce et des taches pigmentées sur la peau, la dysplasie fibreuse représente un des symptômes du syndrome de McCuneAlbright.

L'étiologie moléculaire du syndrome de McCune-Albright implique une mutation somatique de la sous-unité $\alpha$ de la protéine $\mathrm{G}$ (guanine-nucleotidebinding) activant l'adénylyl cyclase $(\mathrm{Gs} \alpha)$ [1]. En effet, le gène $G s \alpha$ est muté dans les tissus de patients atteints du syndrome de McCuneAlbright, de même que dans les lésions osseuses de patients souffrant de dysplasie fibreuse $\left(\mathrm{m} / \mathrm{s} n^{\circ} 7, v o l\right.$. 6 , p. 708) [2]. Ces mutations affectent l'activité de la protéine Gs $\alpha$, conduisant à l'activation constitutive de l'adénylyl cyclase. L'accumulation d'AMPc résultant de l'activation constitutive de la cyclase dans les tissus atteints serait responsable des symptômes associés à la maladie. Il est établi que l'AMPc agit comme second messager dans une cascade de signalisation intracellulaire [3] ; or, les événements moléculaires survenant en aval de l'accumulation d'AMPc dans les tissus atteints res-
Les travaux de Candelière et al., de l'université McGill à Montréal (Québec), publiés récemment dans le New England Journal of Medicine, indiquent que, dans les lésions osseuses de patients atteints de dysplasie fibreuse, le proto-oncogène $c$-fos représente la cible de la voie de signalisation intracellulaire initiée par l'accumulation d'AMPc [4].

Le gène $c$-fos est l'homologue cellulaire de l'oncogène fos des virus murins FBJ et FBR, qui induisent des ostéosarcomes che z la souris. La protéine c-Fos contient un motif d'interaction protéique, la glissière de leucines (leucine zipper), qui lui permet de former des hétérodimères avec différents partenaires, dont les membres de la famille de proto-oncogènes Jun [5]. Sous sa forme hétérodimérique, c-Fos agit comme facteur de transcription et peut se lier à l'ADN à un site spécifique, appelé site AP-1. La protéine c-Fos, avec la complicité de ses différents partenaires, peut donc contrôler l'expression de gènes cibles contenant des sites AP-1 au sein de leur séquence promotrice [6]. D'élégantes expériences ont démontré que la structure de l'os est affectée lorsque l'expression de c-fos est perturbée. L'invalidation du gène par recombinaison homologue provoque, sous forme homozygote, l'inhibition de l'ostéoclastogenèse [7], ce qui conduit à un phénotype d'ostéopétrose $[6,8,9]$. En revanche, une expression élevée de c-fos, chez. des souris transgéniques, provoque des lésions osseuses caractérisées par du tissu fibreux et une structure apparentée à l'os tissé embryonnaire [10]. L'apparence de ces lésions est très similaire à celle des perturbations osseuses observées chez les patients atteints de dysplasie fibreuse, ce qui a conduit l'équipe de McGill à analyser les niveaux d'expression de c-fos dans l'os de ces patients afin de déterminer si une expression anormale de c-fos pourrait être impliquée dans l'étiologie de la maladie.

La technique de l'hybridation in situ comme méthode d'évaluation de l'expression de c-fos a permis de détecter une surexpression de l'ARN messager de c-fos dans tous les échantillons de biopsie osseuse provenant des lésions de huit patients atteints de dysplasie fibreuse [4]. L'expression anormale de la protéine c-Fos a aussi été détectée dans certaines de ces biopsies à l'aide de techniques immunohistochimiques. Quatre échantillons d'os normal n'exprimaient pas c-fos. De plus, aucune expression de c-fos n'a pu être mesurée dans des échantillons de patients atteints d'autres maladies osseuses, tels l'ostéogenèse imparfaite, la maladie de Paget ou le syndrome de Protéus. Les résultats de l'équipe de Montréal démontrent une surexpression de c-fos spécifique des lésions dysplasiques et suggèrent que l'expression anormale de ce protooncogène est responsable des anomalies de développement osseux observées chez les patients atteints de dysplasie fibreuse [4].

L'accumulation d'AMPc induit l'activité de la protéine kinase $A$. Cette voie de signalisation culmine en la phosphorylation de membres de la famille de facteurs de transcription CREB (cAMP response element binding protein) qui se lient à des sites de réponse à l'AMPc ( $c A M P$ response ele ment ou CRE) afin d'activer l'expression des gènes cibles $\left(\mathrm{m} / \mathrm{s} n^{\circ} 11\right.$, vol. 9, p. 1275) [3]. Le promoteur du 
gène $r$-fos contient un site de réponse à l'AMPc (CRE) bien caractérisé [11]. Il est probable que l'accumulation d'AMPc, consécutive à la mutation du gène $G s \alpha$ dans les lésions osseuses affectées, conduise à une expression élevée du proto-oncogène c-fos à travers l'élément CRE du promoteur de c-fos (figure 1).

Ces résultats, qui identifient pour la première fois les événements moléculaires agissant en aval d'une mutation d'une protéine $G$ dans une maladie, n'excluent pas que d'autres mécanismes puissent être responsables de la surexpression de $c$-fos. En effet, une perturbation de la fonction ou de l'expression de n'importe quel gène agissant en amont (tel un récepteur membranaire) ou en aval de $G s \alpha$ conduirait théoriquement à un phénotype similaire. Une direction future de nos travaux consiste en l'analyse systématique du gène $G s \alpha$ dans chacun des échantillons de dysplasie fibreuse. L'absence de mutation du gène $G s \alpha$ dans un échantillon montrant une expression anormale de $c$ fos suggérerait que différents mécanismes moléculaires puissent être impliqués dans la surexpression du proto-oncogène. Les résultats préliminaires de l'équipe de St-Arnaud confortent cette hypothèse.

Une autre voie qu'empruntent nos travaux consiste en l'identification du partenaire de $c$-fos dans l'os. Nous avons démontré que les membres de la famille de proto-oncogènes jun sont exprimés dans l'ostéoblaste [12] et il serait intéressant de sonder les lésions de dysplasie fibreuse afin de mesurer les niveaux d'expression des différents membres de cette famille. Cependant, de nouveaux partenaires de dimérisation de c-Fos ont récemment été identifiés. Le phénotype spécifique de l'os des souris transgéniques exprimant des niveaux élevés du transgène fos dans de nombreux tissus [9] pourrait suggérer l'existence d'un partenaire de dimérisation spécifique à la cellule osseuse. L'équipe de Montréal étudie présentement cette possibilité intéressante.

R.S.A.

1. Chabre O. Une mutation de la protéine Gs $\alpha$ responsable d'hyper- et d'hypo-fonctionnements endocrines. médecine/sciences $1995: 11: 1058$

$m / s n^{\circ} 9$, vol. 11 , seplembre 95

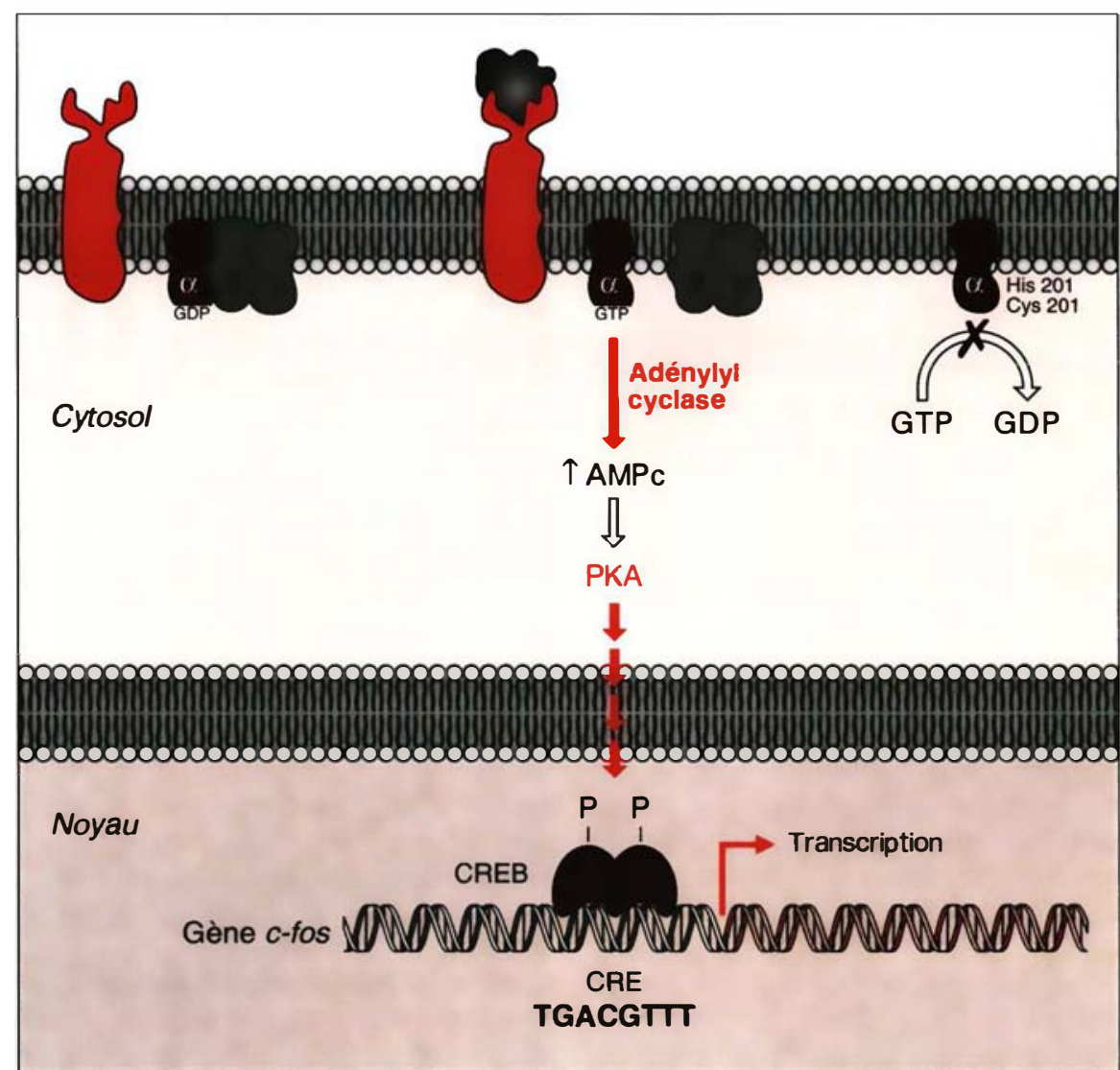

Figure 1. La voie de signalisation intracellulaire impliquant la protéine Gsa stimule l'expression de c-fos à travers l'élément de réponse à l'AMPc ICRE, cAMP response element). La mutation du codon 201 du gène Gs $\alpha$ inhibe l'activité GTPase de la protéine, ce qui conduit à l'activation constitutive de l'adénylyl cyclase. L'accumulation d'AMPc stimule l'activité de la protéine kinase A (PKA), qui induit un cascade de signalisation culminant en la phosphorylation des facteurs de transcription de la famille CREB (cAMP response element binding protein). L'homodimère CREB phosphorylé se lie au site CRE du promoteur de c-fos et stimule l'expression du proto-oncogène.

2. Shender A, Weinstein LS, Sweet DE, Spiegel AM. An activating (ss $\alpha$ mutation is present in fibrous dysplasia of bone in the McCune-Albright syndrome. J Clin Endocinol Metab 1994 ; 79 : 750-5. 3. Lalli E, Sassone-Corsi P. Signal transduction and gene regulation: the nuclear response to cAMP. / Biol Chem $1994 ; 269: 17359-62$

4. Candeliere GA, Glorieux FH, Prud'homme J, St-Arnaud R. Increased expression of the r-fos proto-oncogene in bone from patients with fibrous dysplasia. N Engl J Med 1995 ; 332 : 1546-51.

5 . Blanchard JM. Le proto-oncogène $r$-fos, un "entremetteur moléculaire". médecine/sciences $1992: 8: 455-70$

6. St-Arnaud R. Fonction osseuse : fos et les autres. médecine/sciences $1993 ; 9: 1243-6$

7. Grigoriadis AE, Wang ZQ, Cecchini MG, Hofstetter W, Felix R, Fleisch HA, Wagner EF. c-fos : a key regulator of osteoclast-macrophage lineage determination and bone remodeling Science $1994: 266: 443-8$
8. Johnson RS, Speigelman BM, Papaioannou V Pleiotropic effects of a null mutation in the 6 -fos proto-oncogene. Cell $1992 ; 71: 577-86$

9. Wang ZQ, Ovitt C, Grigoriadis AE, MōhleSteinlein U, Rüther U, Wagner EF. Bone and haematopoietic defects in mice lacking c-fos. Nature $1992 ; 360: 741-5$.

10. Rūther U, Garber C, Komitowski D, Müller R, Wagner EF. Deregulated c-fos expression inter feres with normal bone development in transgenic mice. Nature $1987 ; 325: 412-6$

11. Sassone-Corsi P, Visvader J, Ferland L, Mellon PL, Verma IM. Induction of proto-oncogene fos transcription through the adenylate cyclase pathway: characterization of a cAMP- responsive element. (ienes I)ev $1988: 2$ : 1529-38.

12. Candeliere GA, Prud'homme J, St-Arnaud R Differential expression of fos and jun family members by calcitriol in osteoblastic cells. Mol Endocrinol 1991 ; 5 : 1780-8. 